\title{
Geant spindel cell lipoma atypically located: a case report
}

\begin{abstract}
Spindle cell lipoma is a histologically distinct variant of lipoma characteristically arising in the subcutis of the posterior neck, upper back, or shoulder. They rarely reach a size more than $2-3 \mathrm{cms}$. In this article, we describe a case of a patient with geant spindle cell lipoma localized in atypical location. A 69-year-old male patient consulted because of a soft tissue mass in the right gluteus gradually increasing in size over the past 3 years. Magnetic resonance imaging (MRI) revealed a lipomatous tumor within the right gluteal muscle. The tumor was $10 \mathrm{~cm} \times 7 \mathrm{~cm}$ and was excised completely at surgery. The pathological diagnosis was intramuscular spindle cell lipoma and immunohistochemical study revealed all spindle cells were strongly positive for CD34. Spindle cell lipoma (SCL) is a relatively rare adipocytic neoplasm that classically arise in the trunk, lower extremities, and distal upper extremities of older male patients. Most of them measure less than $2.5 \mathrm{~cm}$. The large and deep seated lipomas represent a real diagnostic and therapeutic challenge as they can be confused with a well-differentiated low grade liposarcoma or other lipomatous neoplasms.
\end{abstract}

Keywords: spindle cell lipoma, gluteal region, geant lipoma
Volume 10 Issue 6 - 2020

\author{
Abdelkader Mizouni,' Taghrid Tlili, ${ }^{2}$ Waad \\ Farhat,' Mohamed Amin Said,' Houssem \\ Ammar,' Leila Jedidi,' Mohamed Ben \\ Mabrouk,' Ali Ben Ali \\ 'Department of Gastrointestinal Surgery, Sahloul Hospital of \\ Sousse, Faculty of medicine of Sousse, University of Sousse, \\ Tunisia \\ 2Pathology department, Farhat Hached hospital of Sousse, \\ Faculty of medicine, University of Sousse,Tunisia
}

Correspondence: Abdelkader Mizouni, Department of Gastrointestinal Surgery, Sahloul Hospital, University of Sousse, Tunisia, Email abdelkaermizouni@gmail.com

Received: November 03, 2020 | Published: November 13, 2020

\section{Introduction}

Lipomatous tumours represent the most common mesenchymal lesions, with an estimated annual incidence of 1 per 1000 inhabitants, and a malignant to benign ratio of about 1 to $100 .{ }^{1}$ Lipomas are slow growing that rarely reach a size more than $2-3 \mathrm{cms}$. Spindle cell lipoma (SCL) is a rare and distinct variant of lipoma (1.5\% of all adipocytic neoplasms) that was first described by Enzinger and Harvey in $1975 .{ }^{2}$ It is easily confused with liposarcoma. ${ }^{3,4}$ It occurs predominantly in men between 45 and 70 years of age and in most cases is found in the subcutaneous tissue of the neck, shoulder or back. ${ }^{5}$ It has also been described in unusual sites, such as the oral cavity, larynx, bronchus, breast, orbit and extremities. ${ }^{6}$ To our knowledge There are few reported cases of geant spindle cell lipoma in the literature. ${ }^{7,8} \mathrm{We}$ report a rare case of geant SCL localized in the gluteal region.

\section{Patient and observation}

A 69-year-old male patient, consulted with complaints of swelling in the right gluteal region gradually increasing in size for 3 years, with no other associated signs. The clinical examination objected a freely mobile large mass of the right gluteal region measuring $7 \mathrm{cmx} 10 \mathrm{~cm}$, soft, non-painful and with no inflammatory signs. There was no history of previous trauma. Magnetic resonance imaging (MRI) revealed a large, well defined mass of the right ischio-rectal pit. It showed high signal on a T2-weighted image (Figure 1), heterogeneous, iso-signal on a T1-weighted image (Figure 2), and a gadolinium-enhanced image (Figure 3), measuring 76-49-108 mm coming into contact with the internal shutter muscle outside, pushing back the anal canal into it without signs of invasion. On the basis of these radiological findings, we suspected a desmoide tumor, or liposarcoma from which the surgical indication was put. The patient was operated on with a single-block excision of the mass without capsular break-in
(Figure 4-6). The surgical suites were simple. A histopathological examination revealed a well circumscribed mass composed of a mixture of mature adipocytes and spindle cells in varying proportions surrounded by a clear fibrous capsule. Neither the lipomatous nor the spindle cell elements of the tumors showed evidence of necrosis or mitotic activity. The immune histochemical analysis, showed that the lesional spindle cells were strongly immunoreactive for CD34 and the results of MDM2 immunostaining were negative. The diagnosis of SCL of the gluteal region was concluded.

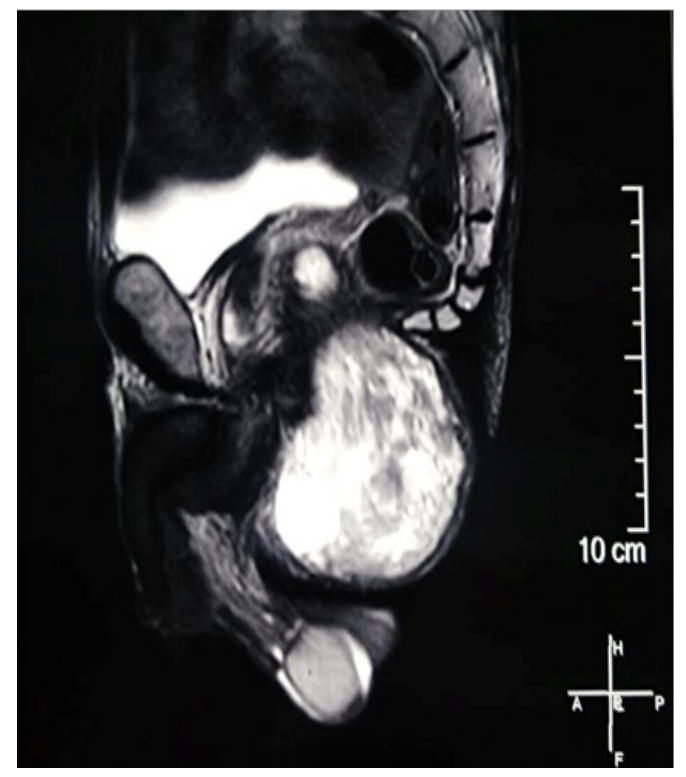

Figure I Sagittal section of Pelvic MRI showing a mass with high signal on a T2 weighted image. 


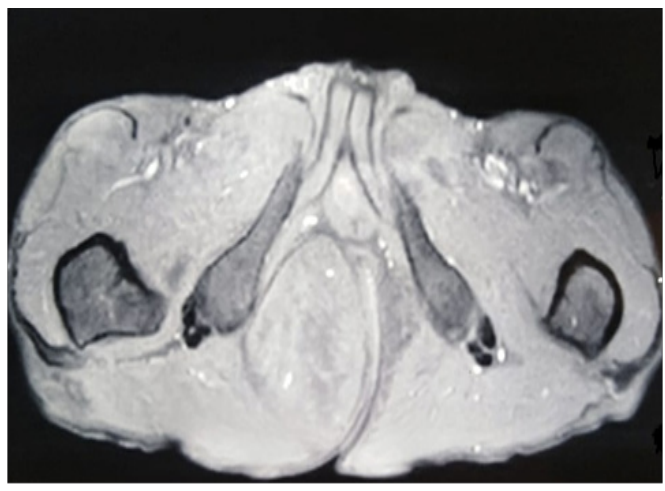

Figure 2 Axial section of Pelvic MRI revealing heterogeneous mass with isosignal on a TI-weighted image.

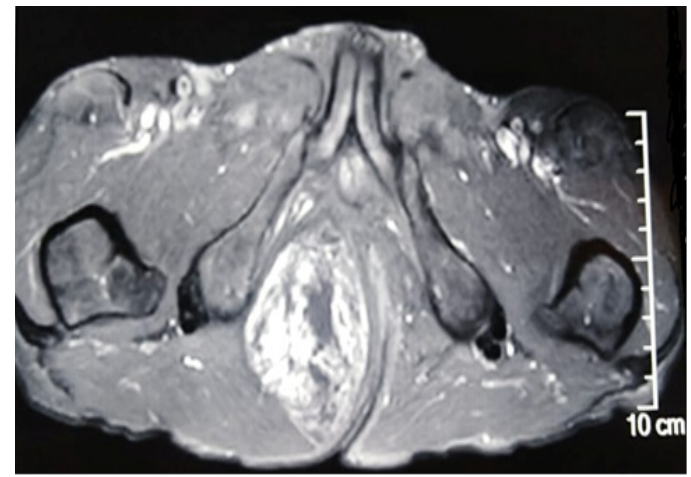

Figure 3 Axial section of Pelvic MRI showing a mass with gadoliniumenhanced on a TI-weighted image.

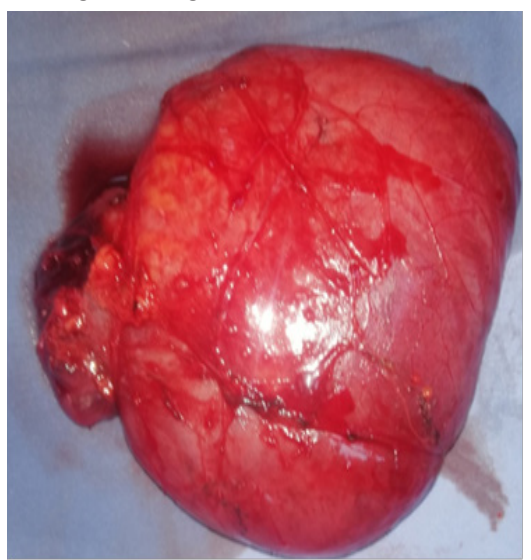

Figure 4 Encapsulated tumor mass.

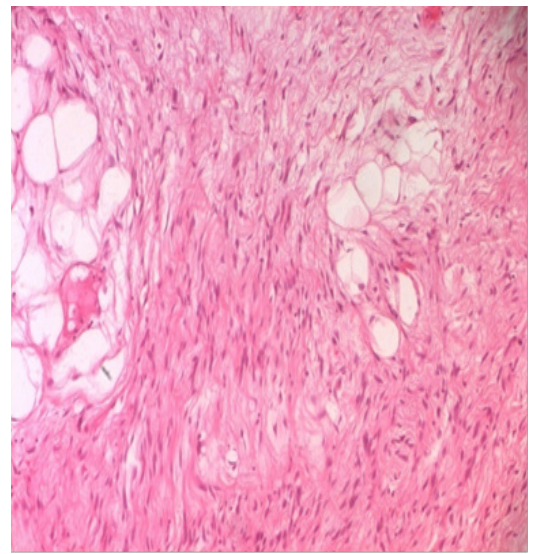

Figure $\mathbf{5}$ Microphotograph of tumor showing an admixture of fat and spindle cells with ropy collagen fibers (H\&Ex 200).

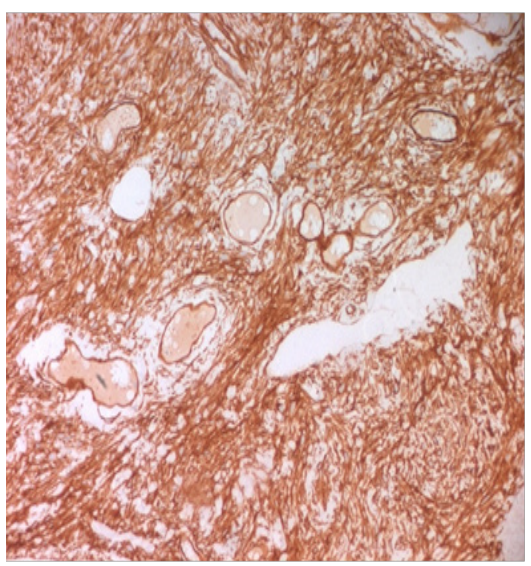

Figure 6 Spindle cells are diffusely and strongly positive to CD34 (x|00).

\section{Discussion}

Spindle cell lipoma (SCL), a benign lipomatous neoplasm that was first described by Enzinger and Harvey in 1975. ${ }^{1}$ It is more common in middle aged men. ${ }^{9}$ The most common locations are at the shoulder and neck. The clinical presentation is not specific, but it is characterized by a slow growing mass or swelling that is asymptomatic and a stability of the size which does not generally exceed $5 \mathrm{~cm} .{ }^{5}$ But in that case range of motion decreases and there may be a functional limitation due to mechanical restriction. Duration in these cases can be from months to years.

A spindle cell lipoma (SCL) is a relatively common tumor that can be challenging to the radiologist, to diagnose, particularly when internal fat content is scant or absent. The role of imagery is found in the differentiation between the malignant forms of fatty tumors and the benign forms; in fact unlike lipoma SCL appearance at MRI is not charactestic and easily misdiagnosed with other soft tissu mass. ${ }^{10}$ In our case the magnetic resonance imaging suspected that the mass was a liposarcoma.

Those of extraordinary size or atypical anatomic location require a proper immunohistological workup to rule out more ominous malignancy. SCL is a relatively rare variant and is a histologically distinct lesion characterized by the replacement of mature fat by a mixture of mature adipocytes, undifferentiated spindle cells and ropelike collagen fibers. ${ }^{11}$ That concorded with the histological result of our case.

By immunohistochemistry, although the spindle cells in spindle cell lipoma are consistently positive for CD34 it is also the same in other lipomatous and nonlipomatous soft tissue tumors; thus, this marker has a small role in the diagnosis of spindle cell lipoma wich is established primarily on histologic findings. ${ }^{10,12}$

Surgical excision is the treatment of choice when the patient is symptomatic. ${ }^{3}$ Marginal excision is described for well-circumscribed lesions and wide excision with free margin is necessary for the infiltrative types, which will help in preventing recurrences. As it has been reported in the litterature the recurrence rate is between 3 and $62.5 \%{ }^{4}$ and occur many years after excision ranging from 14 months to 19 years. ${ }^{3}$ Our patient is in a good clinical condition, free of symptoms during his 21 month follow up.

\section{Conclusion}

Although spindle cell lipoma is a rare, benign adipose tissue tumor with variable morphology, his recognition is crucial to avoid confusion with morphologically similar but more aggressive bland- 
appearing spindle cell tumors. While most SCLs arise in classic sites, diagnosis of those with extraordinary size or atypical location can be challenging to the radiologist, pathologist, or surgeon to diagnose. Knowledge of the common imaging, pathologic features and multiple variant subtypes of SCLs can help to avoid misdiagnosis and guide patient management.

\section{Acknowledgments}

None.

\section{Funding}

None.

\section{Conflicts of interest}

The authors have no conflicts of interest to declare.

\section{References}

1. Enzinger FM, Harvey DA. Spindle cell lipoma. Cancer. 1975;36(5):18521859 .

2. de Bree E, Karatzanis A, Hunt JL, et al. Lipomatous tumours of the head and neck: a spectrum of biological behaviour. Eur Arch Otorhinolaryngol. 2015;272(5):1061-1077.

3. McTighe $\mathrm{S}$, Chernev I. Intramuscular lipoma: a review of the literature. Orthop Rev (Pavia). 2014;6(4):5618.
4. Hombal P. GIANT GLUTEAL LIPOMA: A CASE REPORT AND REVIEW OF LITERATURE. International Journal of Surgery and Medicine. 2015;1(1):26.

5. Eryilmaz M, Yigit T, Ozturk G, et al. Giant spindle cell lipoma of the posterior neck. Dermatol Surg. 2007;33(10):1258-1261.

6. Ud Din N, Zhang P, Sukov WR, et al. Spindle Cell Lipomas Arising at Atypical Locations. Am J Clin Pathol. 2016;146(4):487-495.

7. Mizoshiri N, Shirai T, Terauchi R, et al. Intramuscular spindle cell lipoma of the deltoid: a case report. J Med Case Rep. 2015;9:38.

8. Haas AF, Fromer ES, Bricca GM. Spindle cell lipoma of the scalp: a case report and review. Dermatol Surg. 1999;25(1):68-71.

9. Ko JS, Daniels B, Emanuel PO, et al. Spindle Cell Lipomas in Women: A Report of 53 Cases. Am J Surg Pathol. 2017;41(9):1267-1274.

10. Jelinek JS, Wu A, Wallace M, et al. Imaging of spindle cell lipoma. Clin Radiol. 2020;75(5):396.e15-396.e21.

11. Chen S, Huang H, He S, et al. Spindle cell lipoma: clinicopathologic characterization of 40 cases. Int J Clin Exp Pathol. 2019;12(7):26132621.

12. Lau SK, Bishop JA, Thompson LDR. Spindle cell lipoma of the tongue: a clinicopathologic study of 8 cases and review of the literature. Head Neck Pathol. 2015;9(2):253-259. 